

\title{
Yttrium-90 glass microspheres radioembolization (RE) for biliary tract cancer a large single-center experience
}

Héloïse Bourien, Xavier Palard, Yan Rolland, Fanny Le Du, Luc Beuzit, Thomas Uguen, Samuel Le Sourd, Marc Pracht, Vincent Manceau, Astrid Lièvre, et al.

\section{To cite this version:}

Héloïse Bourien, Xavier Palard, Yan Rolland, Fanny Le Du, Luc Beuzit, et al.. Yttrium-90 glass microspheres radioembolization (RE) for biliary tract cancer a large single-center experience. European Journal of Nuclear Medicine and Molecular Imaging, 2019, 46 (3), pp.669-676. 10.1007/s00259-0184199-5 . hal-01937263

HAL Id: hal-01937263

https://hal-univ-rennes1.archives-ouvertes.fr/hal-01937263

Submitted on 3 Dec 2018

HAL is a multi-disciplinary open access archive for the deposit and dissemination of scientific research documents, whether they are published or not. The documents may come from teaching and research institutions in France or abroad, or from public or private research centers.
L'archive ouverte pluridisciplinaire HAL, est destinée au dépôt et à la diffusion de documents scientifiques de niveau recherche, publiés ou non, émanant des établissements d'enseignement et de recherche français ou étrangers, des laboratoires publics ou privés. 
Héloïse Bourien, Medical Oncology, Centre Eugène Marquis, Rennes, France Xavier Palard, Nuclear Medicine, Centre Eugène Marquis, Rennes, France

Fanny Le Du, Medical Oncology, Centre Eugène Marquis, Rennes, France

Luc Beuzit, Radiology, CHU Pontchaillou, Rennes, France

Thomas Uguen, Hepatology, CHU Pontchaillou, Rennes, France

Samuel Le Sourd, Medical Oncology, Centre Eugène Marquis, Rennes, France

Marc Pracht, Medical Oncology, Centre Eugène Marquis, Rennes, France

Vincent Manceau, Nuclear Medicine, Centre Eugène Marquis, Rennes, France

Astrid Lièvre, Gastroenterology, CHU Pontchaillou, Rennes, France

Karim Boudjema, Hepatobiliary Surgery, CHU Pontchaillou, Rennes, France

Etienne Garin, Nuclear Medicine, Centre Eugène Marquis, Rennes, France

Julien Edeline, Univ Rennes, INSERM, INRA, Centre de Lutte contre le Cancer Eugène Marquis, 
1

2

3

4

5

6

7

8

9

Abstract:

Purpose: Radioembolization (RE) is a promising treatment option for biliary tract cancers (BTC). We report here the largest series to date using this treatment modality.

Methods: We retrospectively studied data from 64 patients treated outside prospective clinical trial at our institution. We studied baseline characteristics as potential prognostic factors. We studied dose delivered to the tumor as predictive factors of outcomes in patients not receiving concomitant chemotherapy.

Results: The Progression-Free Survival and Overall Survival (OS) survival were 7.6 months [95\% Confidence Interval (Cl): 4.6-10.6] and 16.4 months [95\% Cl: 7.8-25.0] in the whole cohort. The factors independently associated with OS in multivariable analysis were the primary localization of ICC (HR=0.27, 95\% Cl: 0.11-0.68, $\mathrm{p}=0.005)$ and a PS>0 (HR=2.21, 95\% Cl: 1.11-4.38, $\mathrm{p}=0.024)$. During follow-up, 12 patients (19\%) underwent surgery following downstaging, with a median OS was 51.9 months. In patients not treated with concomitant chemotherapy ( $n=31)$, OS was significantly higher in patients with a dose delivered to the tumor 260Gy or higher than in patients with a dose delivered to the tumor lower than 260Gy (median 28.2 vs 11.4 months, log-rank $p=0.019$ ).

Conclusion: Our results confirm that RE is a promising treatment modality in BTC. A high proportion of patients could be downstaged to surgery, with promising long term survival. Dose delivered to the tumor correlated with clinical outcomes when chemotherapy was not used concomitantly. 


\section{Introduction}

Intrahepatic cholangiocarcinoma (ICC) has a rising incidence in Western countries $[1,2]$. Other biliary tract cancers (BTC) (hilar, distal cholangiocarcinomas and gall-bladder adenocarcinoma) have a high propensity to metastasize to the liver. In advanced BTC, doublet chemotherapy with cisplatin and gemcitabine became the standard treatment after the results of the ABC-02 study (a phase 3 study showing the superiority of the cisplatin-gemcitabine combination over gemcitabine monotherapy) and a subsequent meta-analysis [3-5].

${ }^{90} \mathrm{Y}$-microspheres radioembolization (RE), also known as selective internal radiation therapy, is applied as a loco-regional treatment for malignant liver disease. Radiolabeled microspheres are administrated via the hepatic arteries, delivering a local radiation dose when reaching the tumor vasculature. Multiple single-center series reported results of RE in BTC [6-17], however the largest published to date included only 46 patients [11]. There is still considerable uncertainty about potential prognostic factors and about the potential preferred population in which this treatment should be applied $[15,18]$. Cucchetti et al suggested that mass-forming and first-line patients had the best prognosis when comparing the results obtained across the different series available, however this hypothesis was not tested in other cohorts [18]. We previously suggested that in first-line patients, concomitant chemotherapy might provide additional benefit [8]. In contrast to what was shown in Hepatocellular Carcinoma $[19,20]$, in a previous analysis limited to first-line patients treated with concomitant chemotherapy, we did not find a threshold dose to predict response to the treatment, as almost all of the patients were responders with a lowest tumoral dose elicting response being 158Gy [21]. The present study reports our experience with RE in non resectable BTC whatever the treatment line, and try to address potential predictive factors for survival or toxicity, in order to better select ideal candidates for the treatment. We also pursued our work on dosimetry focusing on the population not previously studied, namely the population without concomitant chemotherapy. 
Material and methods

\section{Patients}

We retrospectively analyzed data from patients treated at our institution with RE for unresectable BTC (mostly ICC, but also extrahepatic BTC with metastases to the liver). Main inclusion criteria for RE were histologically-proven BTC, with no or limited extrahepatic disease, involvement of $50 \%$ or less of the liver volume by the tumor, adequate liver function (no cirrhosis or Child-Pugh class A cirrhosis, with bilirubin level $\leq 35 \mu \mathrm{mol} / \mathrm{L}$; we extrapolated Child Pugh score to patients without cirrhosis to assess liver function), without elevated pulmonary shunt (with a lung dose higher than 30 Gy), and performance status of 2 or lower. Exclusion criteria were the lack of follow-up available after RE (patient gone to other centers without further information following RE). All patients were discussed during a multidisciplinary team meeting specialized in liver malignancies including hepato-biliary surgeons and radiologists, and their disease were judged unresectable. We included all consecutive patients meeting inclusion criteria, but excluded patients enrolled in prospective clinical trials with RE.

As previously described [8], we defined concomitant chemotherapy as the administration of chemotherapy starting at a maximum of 3 months before RE, without any radiologic assessment of response before RE. When chemotherapy was started 3 months or more before RE, and/or when radiologic evaluation was performed before RE, we used the term induction chemotherapy [8].

Treatment received

The RE therapeutic procedure was performed as previously described [22]. The aim of the diagnostic angiography was to define the best catheter position for right, left, or segmental treatment in order to target the lesion. Percentage of pulmonary shunting and absence of digestive uptake were assessed after ${ }^{99 \mathrm{~m}} \mathrm{Tc}$ macroaggregated albumin was injected selectively in the hepatic artery (185MBq). Planar and SPECT/CT acquisitions were performed. SPECT/CT acquisitions were conducted using the following parameters: window $140 \pm 7.5 \mathrm{KeV} ; 32$ projections; $180^{\circ} ; 128 * 128$; 
30s/projection (Symbia T2 gantry, Siemens). The data was reconstructed using an iterative method (OSEM, 5 iterations, 8 subsets) with CT based attenuation correction and scatter corrections.

Radioembolization was performed 8 to 15 days later at a second angiography, using glass microspheres. We performed only lobar treatment, one in case of unilobar, two in case of bilobar disease, but some patients with anatomical variants could have 3 treatments. Activity administrated was calculated with the aim of administering a dose between 80 and 150 Gy to the targeted liver volume without exceeding a cumulative dose of $30 \mathrm{~Gy}$ to the lungs; however, in case of segmental or bisegmental injection, dose to the segment could be higher than $150 \mathrm{~Gy}$ as previously described [19]. Segmentation (targeted liver and tumor) was performed on SPECT/CT data and not on the angiographic and CT data usually used, as previously described $[19,21,23]$. The doses in the selected volume of interest (VOIs), i.e., tumor, targeted liver, and healthy targeted liver, were calculated using the classic medical internal radiation dose (MIRD) formula, given below:

$$
D_{\text {vol }}(G y)=A_{\text {vol }}(G B q) \times 50 / W_{\text {vol }}(k g)
$$

where $\mathrm{D}_{\mathrm{VOI}}=$ mean dose in the $\mathrm{VOI} ; \mathrm{A}_{\mathrm{VOI}}=$ total activity in the $\mathrm{VOI} ; \mathrm{W}_{\mathrm{VOI}}=$ weight of the $\mathrm{VOI}$ with $\mathrm{W}=$ volume of the VOI x 1.03. The Volumetric Analysis software (Syngo workstation, Siemens) was used for the dosimetric evaluation

Four different chemotherapy regimens were administered, as follows: (1) the modified LV5FU2cisplatin regimen consisted in cisplatin at $50 \mathrm{mg} / \mathrm{m} 2$ on day $1,5 \mathrm{FU}$ bolus at $400 \mathrm{mg} / \mathrm{m} 2$ on day 1 , and $5 F U$ continuous infusion at $2400 \mathrm{mg} / \mathrm{m} 2$ upon 46 hours, cycles repeated every 2 weeks; (2) the GEMOX regimen consisted in gemcitabine $1000 \mathrm{mg} / \mathrm{m} 2$ on day 1 and oxaliplatin $100 \mathrm{mg} / \mathrm{m} 2$ either on day 1 or 2, cycles repeated every 2 weeks; (3) the gemcitabine cisplatin regimen consisted in cisplatin $25 \mathrm{mg} / \mathrm{m} 2$ on day 1 and 8 and gemcitabine $1000 \mathrm{mg} / \mathrm{m} 2$ on day 1 and 8, cycles repeated every 3 weeks or (4) the gemcitabine regimen consisting in gemcitabine $1250 \mathrm{mg} / \mathrm{m} 2$ on day 1,8 and 15 
repeated every 4 weeks. When patients received concomitantly gemcitabine and RE, the dose of gemcitabine was reduced to $300 \mathrm{mg} / \mathrm{m} 2$ for the cycles preceding and after RE, by analogy to the recommended dose for concomitant chemoradiotherapy in pancreatic cancer [24]. The chemotherapy regimen varied across time according to evolution of standards of treatment, according to patients' characteristics and according to some clinicians' preferences when patients were coming from other institutions. Concomitant chemotherapy was administered on the day before or after RE, but not on the same day. A line of chemotherapy is defined by a regimen.

\section{Evaluation}

Toxicity was retrospectively graded using NCI-CTCAE v4. We defined hepatic toxicity as the occurrence or at least one grade worsening of ascites, bilirubin, or encephalopathy, even if these toxicities were reversible. We considered acute hepatic toxicity if these toxicities occurred during the first 3 months following RE, and total hepatic dysfunction whenever these toxicities occurred. We assessed whether hepatic toxicity occurred after intra-hepatic progression of the disease, or if no hepatic progression explained the hepatic dysfunction.

Response was prospectively evaluated by CT scan 6 to 8 weeks after RE, then every 2 to 3 months, using RECIST 1.1 and Choi criteria, as we previously showed that Choi criteria might better predict survival in this context $[25,26]$.

Each analysis was performed with the use of a 2 -sided $\alpha$ level of 0.05 by using the SPSS software v21. The $\chi 2$ or the Fisher tests was used for frequency comparisons. Survival data were analyzed with the Kaplan-Meier method, log-rank test, and Cox regression model. Overall Survival (OS) was the time between first RE and death, Progression-Free Survival (PFS) was the time between first RE and either death or progression according to RECIST 1.1. Survival was not censored at the time of surgery. 


\section{Results}

Between August 2010 and October 2016, 64 patients were treated by RE at our institution. Baseline characteristics of the patients are reported in Table 1. The treatment applied is summarized in Table 2.

Median follow-up was 37.5 months. During follow-up, 45 patients (70\%) experienced progression, and 43 patients (67\%) died. Following RE, in the 62 patients evaluable for response, best responses according to RECIST were Partial Response in 9 (15\%), Stable Disease in 38 (61\%) and Progressive Disease in 15 (24\%). According to Choi criteria, it was Partial Response in 44 (71\%), Stable Disease in 5 (8\%) and Progressive Disease in 13 (21\%). Patients experiencing RECIST progression had worse OS (median 7.5 months), but there was no difference in OS between patients with RECIST Stable Disease (median 28.2 months) and patients with RECIST Partial Response (median 21.5 months). In contrast, Choi evaluation was able to distinguish between patients with Progressive Disease (median 7.5 months), patients with Stable Disease (median 19.1 months) and patients with Partial Response (median 28.2 months) ( $p<0.001)$.

Median PFS for the whole cohort was 7.6 months [95\% Confidence Interval (CI): 4.6-10.6] (Figure 1A). Median PFS was longer for ICC patients than for other BTC, with a median of 9.1 months and 4.9 months respectively $(p=0.009)$. No other parameter was associated with differences in PFS. PFS was 9.5 months [95\% Cl: 7.2-11.9] when RE was included in the first line of treatment vs 5.7 months when it was used as further line, but the difference was not statistically significant $(p=0.49)$. Progression was seen in the treated lesion in 13 patients (20\%), in the liver in 32 patients (50\%) and outside the liver in 32 (50\%). All patients with progression in the treated liver had concomitant progression in both the liver and outside the liver.

Median OS for the whole cohort was 16.4 months [95\% Cl: 7.8-25.0] (Figure 1B). There was a worse survival in patients who had previous biliary stenting (median of 5.5 vs 19.1 months, $p=0.023$ ), in patients with a primary location different from ICC (median of 5.5 vs 19.1 months, $p=0.009$ ) in 
patients with a PS>0 vs PS=0 (median of 9.6 months vs 31.4 months, $p=0.040$ ) and in patients with a tumor progressive after first-line chemotherapy (median of 7.5 vs 20.0 months, $p=0.019$ ). No other parameter was associated with significant difference in OS. Regarding patients with biliary stent, 3 out of $4(75 \%)$ died within 6 months due to hepatic failure without intra-hepatic progression, and one died at 7 months due to multifocal extra hepatic progression. There was a trend toward a worse OS when RE was used in later lines of treatment, with a median OS of 19.9 months when RE was included in the first line, 11.4 months in the second line and 7.5 months in the third line and more $(p=0.10)$. PFS and OS median in different sub-groups are presented in Table 3.

During follow-up, 12 patients (19\%) underwent surgery following downstaging. R0-surgery was obtained in 8 patients (66\%). Major hepatectomy were performed in all cases: two patients underwent right lobectomy, three patients underwent left hepatectomy and 7 patients underwent right hepatectomy (in 4 cases, extended to segment 1). Within 3 months post surgery, 9 patients (75\%) experienced complication; 3 presented pleural effusion, one developed ascites, 4 had hepatic dysfunction and one presented a stroke. 3 patients (25\%) experienced complication of grade 3 or more. Among these 3 patients, two died: one had a massive stroke on postoperative day 9, one developed a severe liver failure due to thrombosis of both the hepatic artery and portal vein. Downstaging to surgery was more frequent in patients treated with RE as part of their first line (10/36 patients, $28 \%)$ and in patients treated with concomitant chemotherapy (10/33 patients, 30\%). Median OS was 51.9 months [95\% $\mathrm{Cl}: 0.0-113.4]$ for patients who underwent a surgical resection vs 15.0 [95\% Cl: 5.3-25.6] for patients who did not ( $p=0.024$, figure 2$)$.

Toxicities during the first 3 months following RE (and in some case concomitant chemotherapy) were reported in Table 4. Toxicity was absent in 25 (39\%) patients. Some form of hepatic dysfunction was seen in 26 (41\%) patients during the follow-up. These hepatic dysfunctions occurred at a median of 7.2 months after RE (range: 1- 35 months), and occurred after intra-hepatic progression in 17 (74\%), after major hepatic surgery in $4(6 \%)$, and with no specific associated factor, and thus considered 
related to RE in 5 ( $22 \%$ of patients with hepatic dysfunction, $8 \%$ of the whole cohort). Three out of 5 have biliary stent. Hepatic dysfunction was seen more frequently in patients with bilobar disease $(17 / 36,47 \%)$, than in patients with unilobar disease $(6 / 28,21 \%)(p=0.033)$, in patients with multifocal disease $(20 / 46,44 \%)$ than in patients with unifocal disease $(3 / 18,17 \%)(p=0.044)$, and was also seen in $6 / 12(50 \%)$ cirrhotic patients vs $17 / 52$ (33\%) non-cirrhotic patients, albeit this difference was not statistically significant $(p=0.26)$. Hepatic dysfunction was associated with worse OS, with a median OS of 10.0 months in case of dysfunction vs 33.8 months in the absence of dysfunction $(p=0.010)$.

We then developed a prognostic model for OS. When entering only baseline characteristics significantly associated with OS in univariable analysis, the factors independently associated with OS in multivariable analysis were the primary localization of ICC (HR=0.27, 95\% Cl: $0.11-0.68, p=0.005)$, a PS $>0 \quad(H R=2.21,95 \% \mathrm{Cl}: 1.11-4.38, p=0.024)$. When considering also variables available after treatment (response criteria and hepatic dysfunction), factor associated with OS were the primary localization of ICC (HR=0.10, 95\% Cl: 0.03-0.31, $\mathrm{p}<0.001)$, a $\mathrm{PS}>1 \quad(\mathrm{HR}=3.16,95 \% \mathrm{Cl}$ : 1.42-7.01, $\mathrm{p}=0.005)$ and Choi response $(\mathrm{HR}=3.22,95 \% \mathrm{Cl}: 1.88-5.53, \mathrm{p}<0.001)$, but not RECIST response or hepatic dysfunction.

We then focused on the ICC population. Median OS and PFS for patients with ICC was respectively 19.1 months [95\% Cl: 9.6-28.6] and 9.1 months [95\% Cl: 6.5-11.6]. In univariable analysis, PS>0 was borderline significant $p=0,059(H R=1,970,95 \% \mathrm{Cl}: 0.97-3.89)$. No parameter was significantly associated with OS, due to limited power.

We finally studied dose delivered to the tumor as a predictor of clinical outcomes. Our previous work focusing on patients treated in first-line with concomitant chemotherapy, we focused here on the population not previously studied, namely the population without concomitant chemotherapy, whatever the line of treatment $(n=31)$. The dose delivered to the tumor did not correlate with RECIST response, with a median of 263Gy in patients with RECIST objective response vs 269Gy in patients without RECIST objective response $(p=1.00)$; however, the dose delivered to the tumor differed with 
1 Choi response, with a median of 280Gy in patients with Choi objective response vs 227Gy in patients

2 without Choi objective response $(p=0.050$, Figure $3 A)$. We defined by Receiver Operating Curve

3 analysis the optimal threshold for dose delivered to the tumor as $260 \mathrm{~Gy}$ as a predictor of Choi

4 objective response. Using this threshold, in the 29 patients evaluable for Choi response, 14/16 (88\%)

5 treated with 260 Gy or higher had objective response vs $5 / 13(39 \%)$ treated with lower than $260 \mathrm{~Gy}$

$6 \quad(p=0.016$, Figure 3B). Overall survival was significantly higher in patients with a dose delivered to the

7 tumor 260Gy or higher than in patients with a dose delivered to the tumor lower than 260Gy

8 (median 28.2 vs 11.4 months, log-rank $\mathrm{p}=0.019, \mathrm{HR}=0.35,95 \% \mathrm{Cl}: 0.14-0.87, \mathrm{p}=0.024$, Figure $3 \mathrm{C}$ ). For

9 the ICC subgroup, OS was also significantly higher in patients with a dose delivered to the tumor

$10260 \mathrm{~Gy}$ or higher than in patients with a dose delivered to the tumor lower than 260Gy (median 28.2

11 vs 11.4 months, log-rank $p=0.018, \mathrm{HR}=0.33,95 \% \mathrm{Cl}: 0.12-0.86, p=0.024)$. 


\section{Discussion}

This series is the largest series published to date reporting results of RE in BTC, and has also a long median follow-up $>3$ years. This series confirms the promising results of RE in this context, with a median OS of 16.4 months overall and 19.9 months in the first-line setting, comparing favorably with results achieved with chemotherapy alone. Important new results are also the identification of potential prognostic factors, the evidence of a high proportion of patients downstaged to surgery, and the evidence of a correlation between dose delivered to the tumor and the response and OS.

Regarding the prognostic factors, our study might help to suggest the optimal population which should be the focus of future studies. The multivariable analysis suggests that ICC are a more favorable setting than other BTC with liver involvement, and finds that the main other prognostic factor was PS. Multifocality of the disease and presence of extrahepatic spread did not seem to be associated with OS, however we clearly selected patients with no major liver involvement $(<50 \%)$ and with limited extra-hepatic spread (mainly limited lymph nodes or small lung lesions). Moreover, we did not find that infiltrative type was significantly associated with worse OS, but this may be related to the low power of the study. Infiltrative type is also quite subjective, and might be difficult to reproduce. We did not show significant difference in PFS or OS between lines of therapies, despite some clear numerical differences (median OS of 19.9 months in first line, 11.4 months in second line and 7.5 months in later lines).

RE was applied in this series in different clinical settings, ranging from first-line with one single but unresectable lesion to third-line with multifocal spread. Our results suggest that application in the first-line setting might be the best one for 2 main reasons. First, we found a better OS in the first-line setting, even if it did not reached statistical significance. Second, and more importantly, the use in first-line setting was associated with a high proportion of downstaging to surgery (28\%), with an impressive achieved median OS of more than 4 years in these operated patients, similar in this initially unresectable population to what is seen in operable patients treated with adjuvant 
chemotherapy in recent clinical trials $[27,28]$. We previously described surgical possibilities following RE $[8,29]$. This high rate of downstaging to surgery and long survival might suggest than RE could be a promising option in neoadjuvant strategies in unresectable but pauci-focal disease and also in borderline-resectable cases, as R1- or resection with margin $<5 \mathrm{~mm}$ were shown to be prognostic in ICC $[30,31]$. Additionally, a single-center study previously showed similar results in patients treated with intra-arterial therapies than with surgery for ICC limited to the liver, suggesting that upfront surgery might not be the only loco-regional treatment option [32]. However, our results suggest that surgery following RE improved the results of RE alone, in patients that can be downstaged. This should be further studied.

Toxicities were as expected with RE and chemotherapy, and mostly of low grade. We did see hepatic dysfunctions, some occurring late after treatment, but in most cases it was associated with intrahepatic progression, and probably more related to the disease than the treatment. Liver dysfunction in the absence of progression was seen in only $8 \%$ of the patients. However, it is important to note the higher incidence of hepatic dysfunction in patients with multifocal disease (44\%) in patients with cirrhosis $(50 \%)$, and in patients with biliary stent ( 3 out of 4 ): these patients are also at higher risk for hepatic dysfunction in case of progressive disease.

We showed that a dose delivered to the tumor of 260Gy was predictive of outcomes in terms of Choi response and OS. This differed from our previous results regarding EASL response in patients treated in first-line with concomitant chemotherapy [21]. At least 2 difference between both studies might explain the discrepancy with our previous work: first, concomitant chemotherapy is likely to decrease the threshold for response, as we previously showed synergy between chemotherapy and ${ }^{90} \mathrm{Y}$ in cholangiocarcinoma cell lines [33]. Indeed, in our previous work we were able to see responses with doses as low as $158 \mathrm{~Gy}$ in case of concomitant chemotherapy, suggesting very high sensitivity to radiation in this setting. Second, we used here Choi response criteria, which we previously showed to be better to evaluate response than RECIST criteria in patients treated with RE for ICC [26]. While 
other have suggested EASL or mRECIST to be feasible in ICC, it might be less reproducible than Choi in ICC, due to the peripheral and late pattern of enhancement, differing from Hepatocellular carcinoma [10]. Finally, our results here are in lines with accumulating evidence of the importance of dosimetry in RE [34].

Our study has some limitations. This is a single retrospective institution experience, thereby making definitive recommendations difficult. Second, due to the relative rarity of unresectable biliary tract cancer, our population is heterogeneous. This heterogeneity includes both patients' characteristics (primary localization of BTC, size of tumor, presence of extra hepatic disease) and modality of treatment (line of chemotherapy, induction or concomitant chemotherapy, surgical intervention).

In conclusion, this large series confirms the promising activity of RE in ICC. Prospective studies are being carried on. We recently reported the promising results of MISPHEC, a multicentric phase 2 study of combination of RE and chemotherapy in first-line [35], and are awaiting further follow-up for future publication of the results. Another study is comparing RE with transarterial chemoembolization [36]. The SIRCCA phase 3 trial (clinicaltrials.gov identifier NCT02807181) is currently randomizing patients with non-resectable ICC to either chemotherapy alone or RE followed by chemotherapy. This study will have sufficient power to clearly demonstrate the role of RE in advanced disease. Downstaging strategies in borderline cases should also be considered in future studies, as well as proper consideration of dosimetry. 
1 Funding: This work was supported in part by a grant from the French National Agency for Research

2 called "Investissements d'Avenir" nANR-11-LABX-0018-01

3

4 Conflict of interests: Etienne Garin, Yan Rolland and Julien Edeline are consultant for BTG,

5 manufacturer of glass microspheres.

6

7 Ethical approval: All procedures performed in studies involving human participants were in

8 accordance with the ethical standards of the institutional and/or national research committee and

9 with the 1964 Helsinki declaration and its later amendments or comparable ethical standards. For this type of study formal consent is not required. 


\section{References}

1. Everhart JE, Ruhl CE. Burden of digestive diseases in the United States Part III: Liver, biliary tract, and pancreas. Gastroenterology. 2009;136:1134-44.

2. Razumilava N, Gores GJ. Cholangiocarcinoma. Lancet Lond Engl. 2014;383:2168-79.

3. Valle J, Wasan H, Palmer DH, Cunningham D, Anthoney A, Maraveyas A, et al. Cisplatin plus gemcitabine versus gemcitabine for biliary tract cancer. N Engl J Med. 2010;362:1273-81.

4. Okusaka T, Nakachi K, Fukutomi A, Mizuno N, Ohkawa S, Funakoshi A, et al. Gemcitabine alone or in combination with cisplatin in patients with biliary tract cancer: a comparative multicentre study in Japan. Br J Cancer. 2010;103:469-74.

5. Valle JW, Furuse J, Jitlal M, Beare S, Mizuno N, Wasan H, et al. Cisplatin and gemcitabine for advanced biliary tract cancer: a meta-analysis of two randomised trials. Ann Oncol. 2014;25:391-8.

\section{Mosconi C, Gramenzi A, Ascanio S, Cappelli A, Renzulli M, Pettinato C, et al. Yttrium-90} radioembolization for unresectable/recurrent intrahepatic cholangiocarcinoma: a survival, efficacy and safety study. Br J Cancer. 2016;115:297-302.

\section{Soydal C, Kucuk ON, Bilgic S, Ibis E. Radioembolization with (90)Y resin microspheres for} intrahepatic cholangiocellular carcinoma: prognostic factors. Ann Nucl Med. 2016;30:29-34.

8. Edeline J, Du FL, Rayar M, Rolland Y, Beuzit L, Boudjema K, et al. Glass Microspheres 90Y Selective Internal Radiation Therapy and Chemotherapy as First-Line Treatment of Intrahepatic Cholangiocarcinoma. Clin Nucl Med. 2015;40:851-5.

9. Filippi L, Pelle G, Cianni R, Scopinaro F, Bagni O. Change in total lesion glycolysis and clinical outcome after (90)Y radioembolization in intrahepatic cholangiocarcinoma. Nucl Med Biol. 2015;42:59-64. 
2 criteria in solid tumors and European Association for The Study of the Liver criteria using delayed-

3 phase imaging at an early time point predict survival in patients with unresectable intrahepatic cholangiocarcinoma following yttrium-90 radioembolization. J Vasc Interv Radiol JVIR. 2014;25:25665. Radioembolization for Intrahepatic Cholangiocarcinoma: Safety, Response, and Survival Analysis. J Vasc Interv Radiol. 2013;24:1227-34.

12. Hoffmann R-T, Paprottka PM, Schön A, Bamberg F, Haug A, Dürr E-M, et al. Transarterial Hepatic Yttrium-90 Radioembolization in Patients with Unresectable Intrahepatic Cholangiocarcinoma: Factors Associated with Prolonged Survival. Cardiovasc Intervent Radiol. 2012;35:105-16. Intrahepatic Cholangiocarcinoma: A Preliminary Assessment of This Novel Treatment Option. Ann Surg Oncol. 2010;17:484-91. microspheres for unresectable and failed first-line chemotherapy intrahepatic cholangiocarcinoma: preliminary results. J Cancer Res Clin Oncol. 2017;143:481-9.

Overall Survival of Patients with Unresectable Intrahepatic Cholangiocarcinoma Treated by Means of Yttrium-90 Radioembolization: Results in Therapy-Naïve Patients. Cardiovasc Intervent Radiol. 2018; 16. Swinburne NC, Biederman DM, Besa C, Tabori NE, Fischman AM, Patel RS, et al. 
Evaluation Criteria in Solid Tumors 1.1 Imaging Response and Survival. Cancer Biother Radiopharm. 2017;32:161-8.

17. Shaker TM, Chung C, Varma MK, Doherty MG, Wolf AM, Chung MH, et al. Is there a role for Ytrrium-90 in the treatment of unresectable and metastatic intrahepatic cholangiocarcinoma? Am J Surg. 2018;215:467-70.

18. Cucchetti A, Cappelli A, Mosconi C, Zhong J-H, Cescon M, Pinna AD, et al. Improving patient selection for selective internal radiation therapy of intra-hepatic cholangiocarcinoma: A metaregression study. Liver Int Off J Int Assoc Study Liver. 2017;37:1056-64.

\section{Garin E, Lenoir L, Edeline J, Laffont S, Mesbah H, Porée P, et al. Boosted selective internal} radiation therapy with 90Y-loaded glass microspheres (B-SIRT) for hepatocellular carcinoma patients: a new personalized promising concept. Eur J Nucl Med Mol Imaging. 2013;40:1057-68.

20. Garin E. Radioembolisation of hepatocellular carcinoma patients using ${ }^{90} \mathrm{Y}$-labelled microspheres: towards a diffusion of the technique? Eur J Nucl Med Mol Imaging. 2011;38:2114-6.

21. Manceau V, Palard X, Rolland Y, Pracht M, Le Sourd S, Laffont S, et al. A MAA-based dosimetric study in patients with intrahepatic cholangiocarcinoma treated with a combination of chemotherapy and90Y-loaded glass microsphere selective internal radiation therapy. Eur J Nucl Med Mol Imaging. 2018;

22. Salem R, Lewandowski RJ, Gates VL, Nutting CW, Murthy R, Rose SC, et al. Research reporting standards for radioembolization of hepatic malignancies. J Vasc Interv Radiol JVIR. 2011;22:265-78.

23. Garin E, Lenoir L, Rolland $Y$, Laffont $S$, Pracht $M$, Mesbah $H$, et al. Effectiveness of quantitative MAA SPECT/CT for the definition of vascularized hepatic volume and dosimetric approach: phantom validation and clinical preliminary results in patients with complex hepatic vascularization treated with yttrium-90-labeled microspheres. Nucl Med Commun. 2011;32:1245-55. 
24. Zhu C-P, Shi J, Chen Y-X, Xie W-F, Lin Y. Gemcitabine in the chemoradiotherapy for locally advanced pancreatic cancer: a meta-analysis. Radiother Oncol J Eur Soc Ther Radiol Oncol. 2011;99:108-13.

25. Eisenhauer EA, Therasse P, Bogaerts J, Schwartz LH, Sargent D, Ford R, et al. New response evaluation criteria in solid tumours: revised RECIST guideline (version 1.1). Eur J Cancer Oxf Engl 1990. 2009;45:228-47.

26. Beuzit L, Edeline J, Brun V, Ronot M, Guillygomarc'h A, Boudjema K, et al. Comparison of Choi criteria and Response Evaluation Criteria in Solid Tumors (RECIST) for intrahepatic cholangiocarcinoma treated with glass-microspheres Yttrium-90 selective internal radiation therapy (SIRT). Eur J Radiol. 2016;85:1445-52.

27. Primrose J et al. Adjuvant capecitabine for biliary tract cancer: The BILCAP randomized study. J Clin Oncol ASCO Meet Abstr. 2017;35:Abstr 4006.

28. Edeline J et al. Adjuvant GEMOX for biliary tract cancer: updated relapse-free survival and first overall survival results of the randomized PRODIGE 12-ACCORD 18 (UNICANCER GI) phase III trial. ESMO Conf. 2017;Abstr LBA 29.

29. Rayar M, Sulpice L, Edeline J, Garin E, Levi Sandri GB, Meunier B, et al. Intra-arterial Yttrium-90 Radioembolization Combined with Systemic Chemotherapy is a Promising Method for Downstaging Unresectable Huge Intrahepatic Cholangiocarcinoma to Surgical Treatment. Ann Surg Oncol. 2015;

30. Spolverato G, Yakoob MY, Kim Y, Alexandrescu S, Marques HP, Lamelas J, et al. The impact of surgical margin status on long-term outcome after resection for intrahepatic cholangiocarcinoma. Ann Surg Oncol. 2015;22:4020-4028. 

margins on outcome in patients with intrahepatic cholangiocarcinoma: a multicenter study by the AFC-IHCC-2009 study group. Ann Surg. 2011;254:824-830. 32. Wright GP, Perkins S, Jones H, Zureikat AH, Marsh JW, Holtzman MP, et al. Surgical Resection Does Not Improve Survival in Multifocal Intrahepatic Cholangiocarcinoma: A Comparison of Surgical Resection with Intra-Arterial Therapies. Ann Surg Oncol. 2018;25:83-90.

33. Edeline J, Coulouarn C, Crouzet L, Pracht M, Lepareur N, Clément B, et al. Gemcitabine and Oxaliplatin, but Not Sorafenib or Paclitaxel, Have a Synergistic Effect with Yttrium-90 in Reducing Hepatocellular Carcinoma and Cholangiocarcinoma Cell Line Viability. J Vasc Interv Radiol JVIR. 2015;26:1874-1878.e2.

34. Garin E, Rolland Y, Laffont S, Edeline J. Clinical impact of (99m)Tc-MAA SPECT/CT-based dosimetry in the radioembolization of liver malignancies with (90)Y-loaded microspheres. Eur J Nucl Med Mol Imaging. 2016;43:559-75.

35. Edeline J et al. Selective Internal Radiation Therapy (SIRT) with Yttrium-90-glass-microspheres plus chemotherapy in first-line treatment of advanced cholangiocarcinoma (MISPHEC study). ESMO Conf. 2017;Abstr 771P. radiotherapy (SIRT) versus transarterial chemoembolization (TACE) for the treatment of intrahepatic cholangiocellular carcinoma (CCC): study protocol for a randomized controlled trial. Trials. 2014;15:311. 
2 Table 1: Baseline characteristics of the patients

\begin{tabular}{|c|c|}
\hline & Whole cohort $(n=64)$ \\
\hline Gender & Male: 37 (58\%) / Female: 27 (42\%) \\
\hline Performance Status & $\begin{array}{l}\text { 0: } 33(52 \%) \\
\text { 1: } 24(38 \%) \\
\text { 2: } 1(2 \%) \\
\text { Unknown: } 6(9 \%)\end{array}$ \\
\hline Primary tumor location & $\begin{array}{l}\text { Intrahepatic: } 57(89 \%) \\
\text { Hilar: } 6(9 \%) \\
\text { Extra hepatic: } 1(2 \%)\end{array}$ \\
\hline Extra hepatic metastases & $10(16 \%)$ \\
\hline Multifocal (>1 lesion) liver disease & $46(72 \%)$ \\
\hline Bilobar liver disease & $36(56 \%)$ \\
\hline $\begin{array}{l}\text { Maximal diameter of the largest lesion, } \mathrm{mm} \\
\text { median (range) }\end{array}$ & $77(14-182)$ \\
\hline Portal vein (main or branch) thrombosis & $11(17 \%)$ \\
\hline Infiltrative tumor & $30(47 \%)$ \\
\hline $\begin{array}{l}\text { CA19.9, median UI/L (range) ( } \mathrm{n}=58 \text { with available } \\
\text { data) }\end{array}$ & $36(0-5149)$ \\
\hline Underlying cirrhosis & $12(19 \%)$ \\
\hline $\begin{array}{l}\text { Child score (calculated for cirrhotic and non- } \\
\text { cirrhotic patients) }\end{array}$ & $\begin{array}{l}\text { 5: } 57(89 \%) \\
6: 7(11 \%)\end{array}$ \\
\hline Albumin, $\mathrm{g} / \mathrm{L}$ median (range) & $40(19-48)$ \\
\hline Total Bilirubin, $\mu \mathrm{mol} / \mathrm{L}$ median (range) & $11.7(2.1-41.9)$ \\
\hline
\end{tabular}




\begin{tabular}{|l|l|}
\hline Biliary stent & $4(6 \%)$ \\
\hline Previous liver surgery & $15(23 \%)$ \\
\hline Previous line of chemotherapy & $0: 36(56 \%)$ \\
& $1: 23(36 \%)$ \\
& $>1: 4(8 \%)$ \\
\hline
\end{tabular}

1

2

3 
Table 2: Characteristics of the treatment applied

\begin{tabular}{|l|l|}
\hline Chemotherapy included in the same line as RE & $\begin{array}{l}\text { None: } 17(27 \%) \\
\text { Induction: } 14 \text { (22\%) } \\
\text { Concomitant: } 33 \text { (52\%) }\end{array}$ \\
\hline Type of chemotherapy used & Gemcitabine-platinum: 30 (47\%) \\
& LV5FU2-cisplatin: 14 (22\%) \\
\hline Number of RE procedures & Gemcitabine alone: 3 (5\%) \\
\hline Activity administrated, GBq, median (range) & $1: 44(69 \%)$ \\
\hline Tumoral Dose, Gy, median (range) & $2: 17(27 \%)$ \\
\hline Targeted Liver Dose, Gy, median (range) & $3: 3(5 \%)$ \\
\hline Non-tumoral Liver Dose, Gy, median (range) & $85(0-143)$ \\
\hline
\end{tabular}

2

3 
Table 3: median PFS and OS in different subgroups

\begin{tabular}{|c|c|c|c|c|c|}
\hline \multicolumn{2}{|l|}{ Parameter } & Median PFS & $p$ & Median OS & $p$ \\
\hline \multicolumn{2}{|l|}{ Whole cohort } & 7.6 & & 16.4 & \\
\hline \multirow{3}{*}{$\begin{array}{l}\text { Chemotherapy } \\
\text { during the line } \\
\text { of RE }\end{array}$} & No & 6.7 & \multirow{3}{*}{0.90} & 11.4 & \multirow{3}{*}{0.37} \\
\hline & Induction & 3.5 & & 10.1 & \\
\hline & Concomitant & 9.5 & & 19.9 & \\
\hline \multirow{3}{*}{ Line of } & 1 & 9.5 & \multirow{3}{*}{0.38} & 19.9 & \multirow{3}{*}{0.10} \\
\hline & 2 & 6.0 & & 11.4 & \\
\hline & $>2$ & 5.5 & & 7.5 & \\
\hline \multirow{2}{*}{ Cirrhosis } & No & 6.2 & \multirow{2}{*}{0.90} & 16.4 & \multirow{2}{*}{0.47} \\
\hline & Yes & 10.1 & & 11.4 & \\
\hline \multirow{2}{*}{ Primary Site } & Intrahepatic & 9.1 & \multirow{2}{*}{0.009} & 19.1 & \multirow{2}{*}{0.009} \\
\hline & Other & 4.9 & & 5.5 & \\
\hline \multirow[t]{2}{*}{ Extrahepatic } & No & 7.7 & \multirow{2}{*}{0.25} & 15.0 & \multirow{2}{*}{0.82} \\
\hline & Yes & 5.6 & & 21.5 & \\
\hline \multirow{2}{*}{ Biliary Stent } & No & 8.0 & \multirow{2}{*}{0.064} & 19.1 & \multirow{2}{*}{0.023} \\
\hline & Yes & 4.8 & & 5.5 & \\
\hline \multirow[t]{2}{*}{ Multifocal } & No & 9.5 & \multirow{2}{*}{0.15} & 11.4 & \multirow{2}{*}{0.34} \\
\hline & Yes & 6.0 & & 16.4 & \\
\hline \multirow{2}{*}{ Bilobar Disease } & No & 8.9 & \multirow{2}{*}{0.16} & 11.4 & \multirow{2}{*}{0.96} \\
\hline & Yes & 6.2 & & 20.0 & \\
\hline \multirow{2}{*}{$\begin{array}{l}\text { Portal Vein } \\
\text { Thrombosis }\end{array}$} & No & 6.2 & \multirow{2}{*}{0.63} & 15.0 & \multirow{2}{*}{0.21} \\
\hline & Yes & 10.1 & & 37.4 & \\
\hline \multirow{3}{*}{$\begin{array}{l}\text { Infiltrative } \\
\text { tumor }\end{array}$} & No & 6.7 & \multirow{3}{*}{0.46} & 19.9 & \multirow{3}{*}{0.57} \\
\hline & & & & 107 & \\
\hline & Yes & 7.6 & & 10.2 & \\
\hline
\end{tabular}




\begin{tabular}{|l|l|l|l|l|l|}
\hline \multirow{3}{*}{ PS } & 0 & 9.1 & \multirow{2}{*}{0.14} & 31.4 & \multirow{2}{*}{0.040} \\
\cline { 2 - 3 } & $>0$ & 6.2 & & 9.6 & \\
\hline \multirow{2}{*}{ Child } & A5 & 7.7 & \multirow{2}{*}{0.60} & 16.4 & \multirow{2}{*}{0.38} \\
\cline { 2 - 3 } & A6 & 6.0 & & 11.4 & \\
\cline { 2 - 3 } & & & & & \\
\hline
\end{tabular}

1 
1 Table 4: Incidence of adverse events related to RE:

\begin{tabular}{|l|l|l|l|l|}
\hline Adverse Event & Grade 1 & Grade 2 & Grade 3 & Grade 4 \\
\hline Fatigue & $7(11 \%)$ & $13(20 \%)$ & $10(16 \%)$ & 0 \\
\hline Liver pain & $3(5 \%)$ & $10(16 \%)$ & $6(9 \%)$ & 0 \\
\hline Nausea & 0 & $1(2 \%)$ & $2(3 \%)$ & 0 \\
\hline Vomiting & 0 & $1(2 \%)$ & 0 & 0 \\
\hline Vascular event & 0 & 0 & 1 & 0 \\
\hline Hepatic failure & 0 & $2(3 \%)$ & $2(3 \%)$ & 0 \\
\hline
\end{tabular}

2 
Figures:

2 Figure 1: A: Progression-Free Survival and B: Overall Survival for the whole cohort

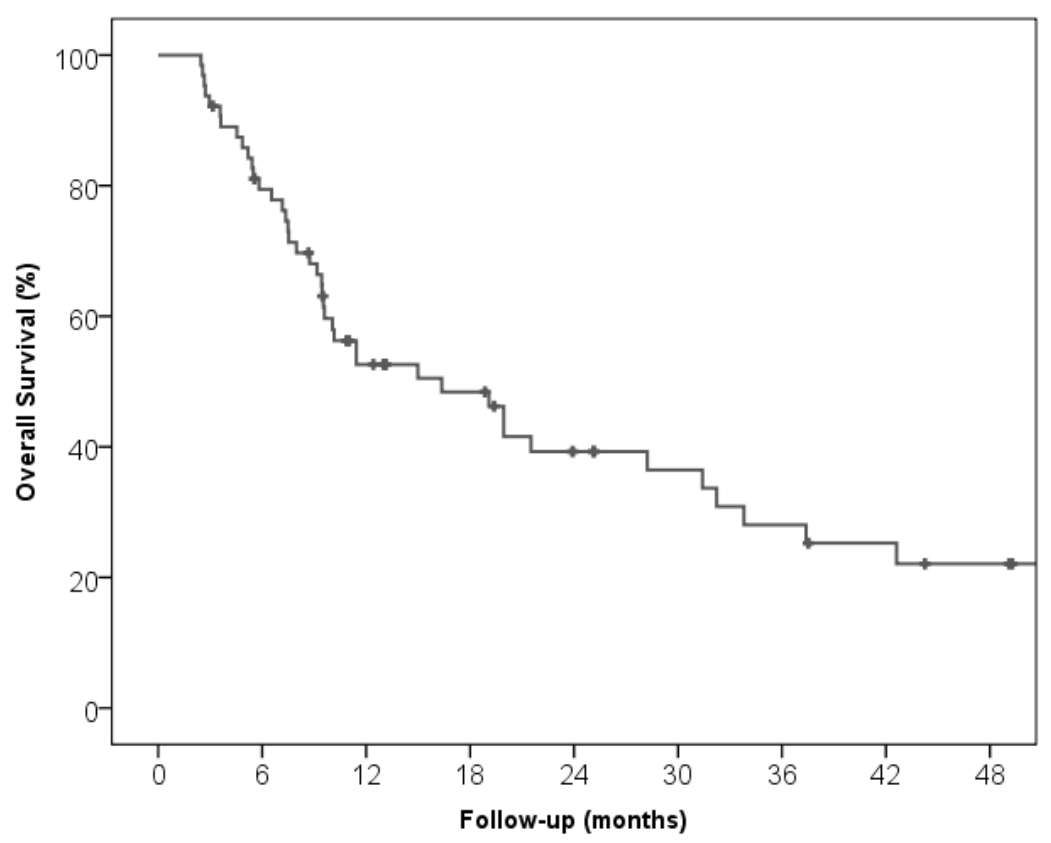

3

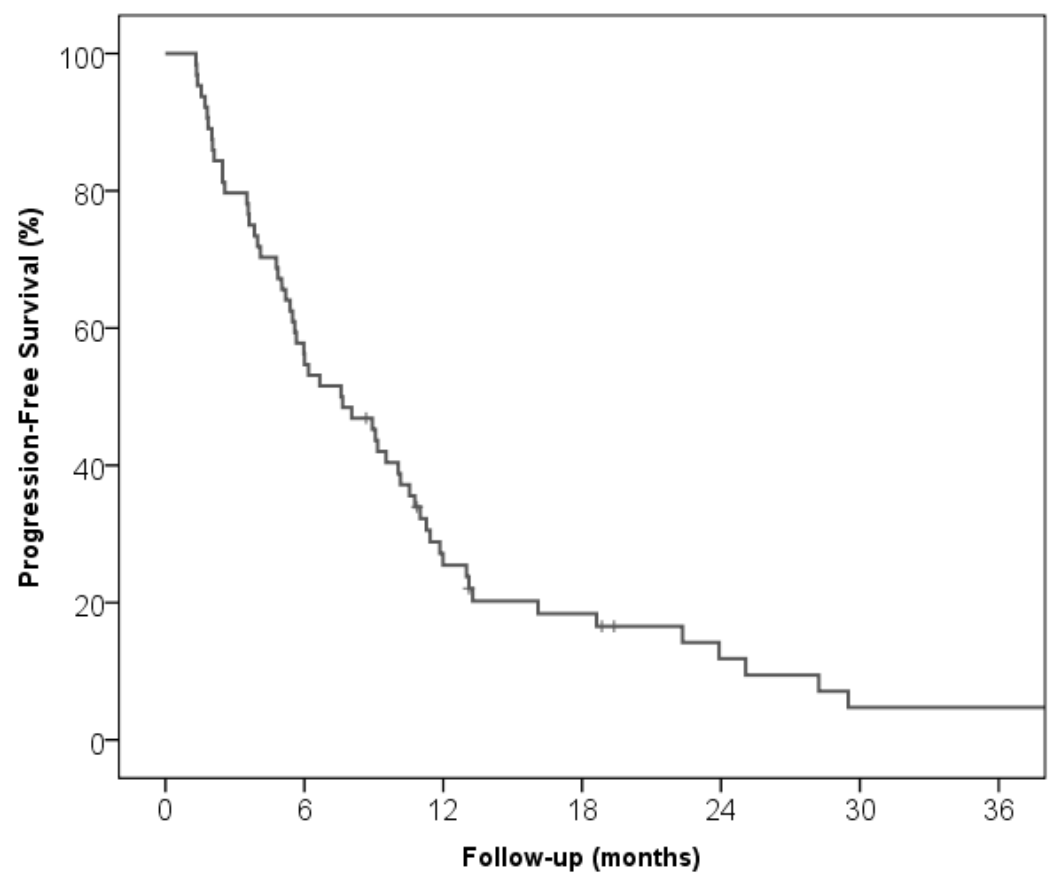

5

6

B 
1 Figure 2: Overall Survival in patients according to resection following RE.

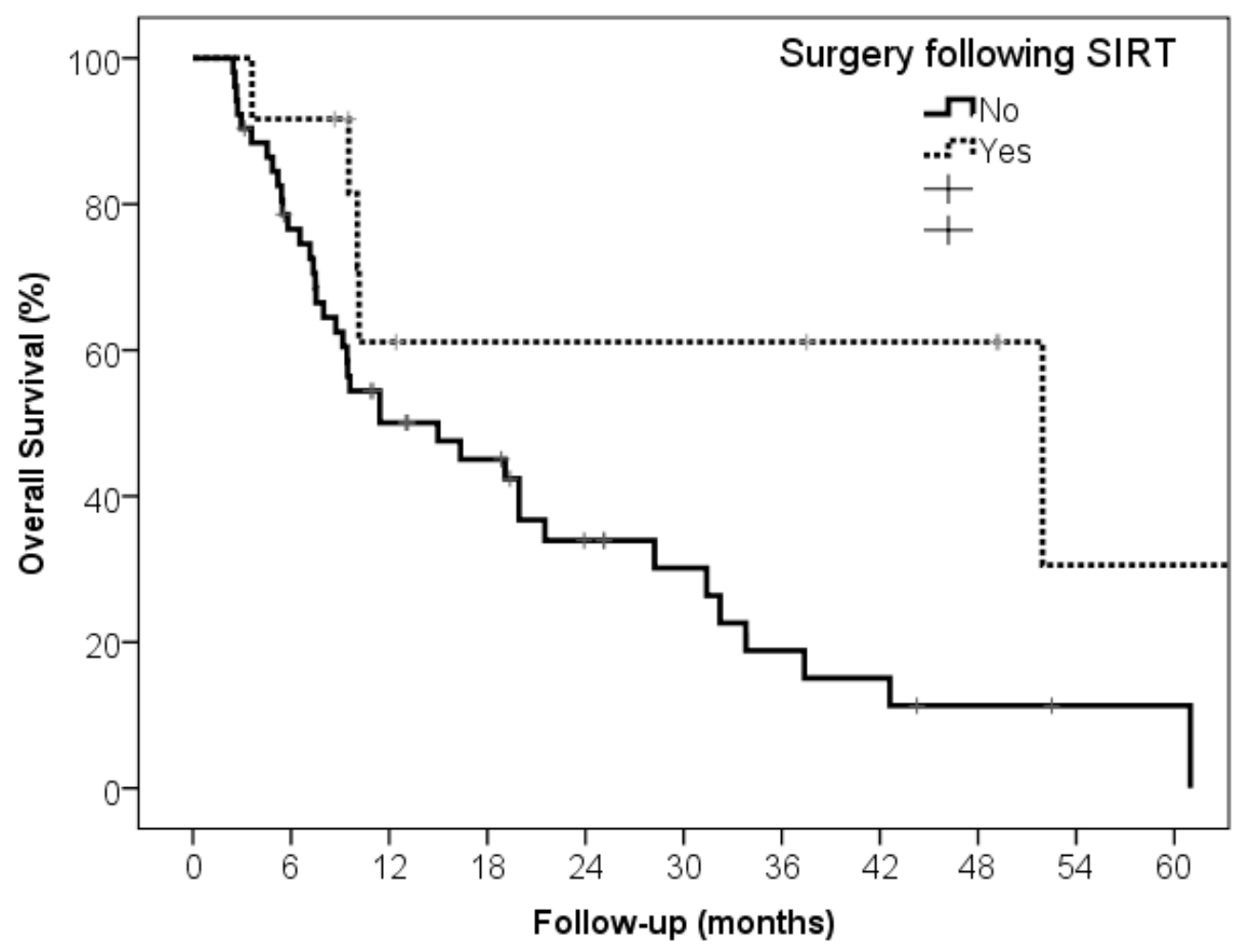

2 3 4 5 6 7 8 9 
1 Figure. 3: Dosimetric analysis of patients treated without concomitant chemotherapy. A: Dose 2 delivered to the tumor in patients with or without Choi response. B: Response rate assessed by Choi 3 criteria according to the dose delivered to the tumor. C: Overall survival according to the dose 4 delivered to the tumor

5

a

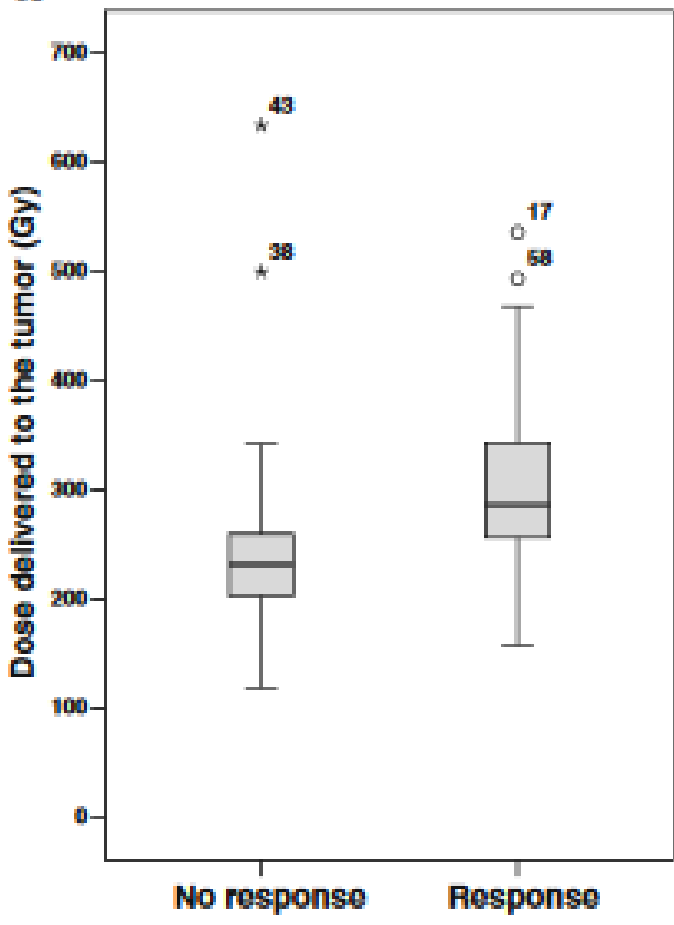

Objective Response by Chol b

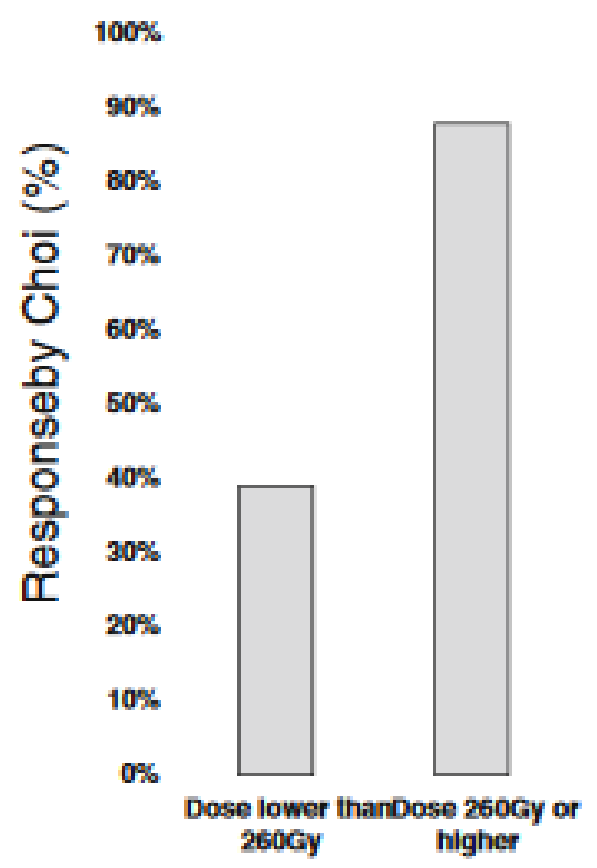




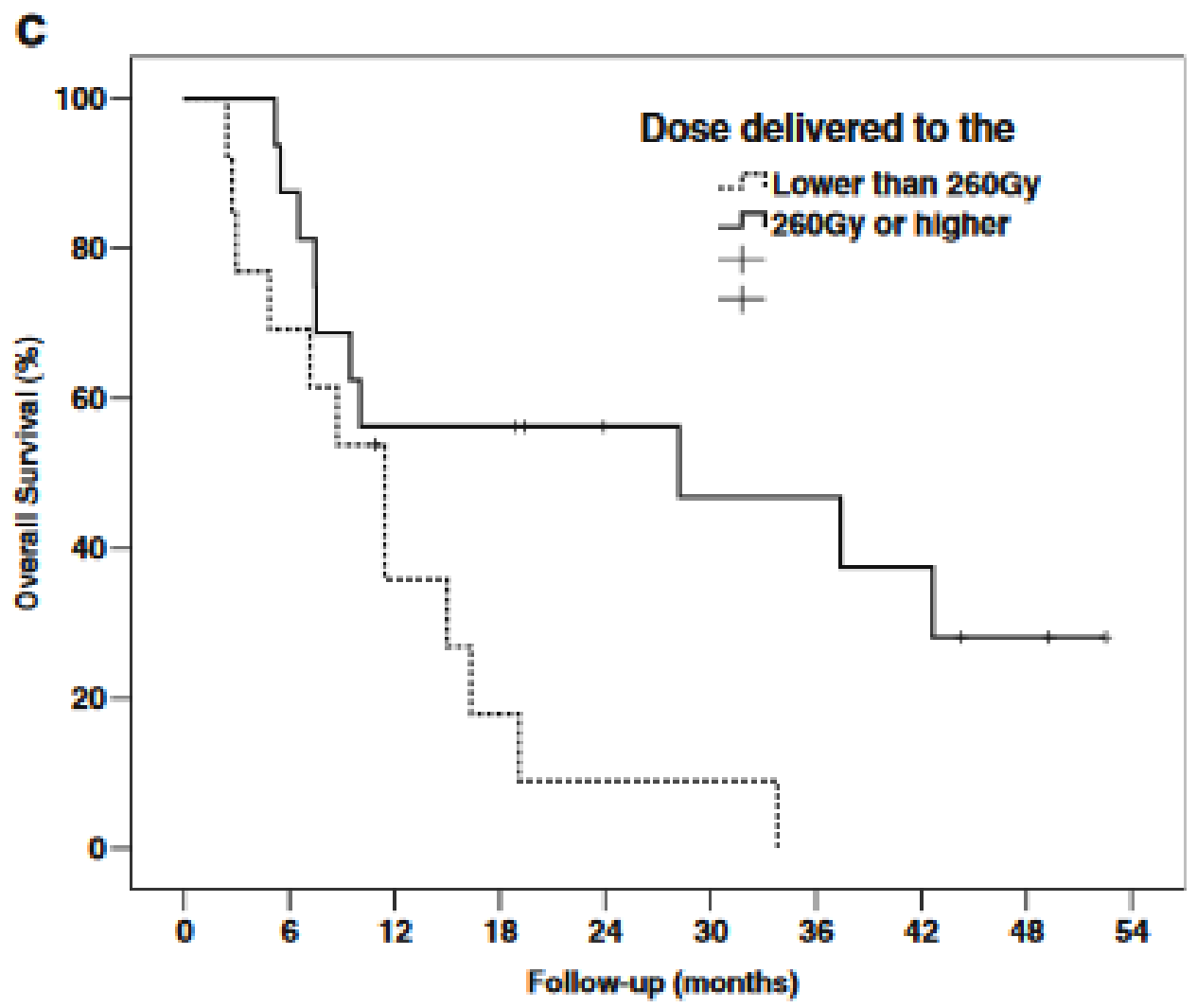

1

2 